\title{
Investigating the effect of cosmic opacity on standard candles
}

\author{
J. $\mathrm{Hu}^{1}, \mathrm{H} . \mathrm{Yu}^{1}$ and F. Y. Wang ${ }^{1,2 *}$ \\ 1 School of Astronomy and Space Science, Nanjing University, Nanjing 210093, China \\ ${ }^{2}$ Key Laboratory of Modern Astronomy and Astrophysics (Nanjing University), Ministry \\ of Education, Nanjing 210093, China \\ fayinwang@nju.edu.cn
}

\begin{abstract}
Standard candles can probe the evolution of dark energy in a large redshift range. But the cosmic opacity can degrade the quality of standard candles. In this paper, we use the latest observations, including type Ia supernovae (SNe Ia) from JLA sample and Hubble parameters, to probe the opacity of the universe. In order to avoid the cosmological dependence of SNe Ia luminosity distances, a joint fitting of the SNe Ia light-curve parameters, cosmological parameters and opacity is used. In order to explore the cosmic opacity at high redshifts, the latest gamma-ray bursts (GRBs) are used. At high redshifts, cosmic reionization process is considered. We find that the sample supports an almost transparent universe for flat $\Lambda$ CDM and XCDM models. Meanwhile, free electrons deplete photons from standard candles through the (inverse) Compton scattering, known as an important component of opacity. This Compton dimming may paly an important role in future supernova surveys. From analysis, we find that about a few percent cosmic opacity is caused by Compton dimming in the two models, which can be correctable.
\end{abstract}

Subject headings: cosmology: theory - distance scale

\section{Introduction}

In 1998, the accelerating expansion of the universe was discovered by measuring the relation between redshift and distance of SNe Ia (Riess et al. 1998; Perlmutter et al. 1999). The physical origin of accelerating is still debated. The term "dark energy" is put forward to explain the accelerating universe. Meanwhile, the modification of equations governing gravity can also explain the acceleration of the universe (i.e., Capozziello 2002). Besides SNe Ia, other observations, such as cosmic microwave background (CMB) (i.e., Spergel et al. 2003), 
baryonic acoustic oscillations (BAO) (i.e., Eisenstein et al. 2005), Hubble parameters (i.e., Jimenez et al. 2003), and gamma-ray bursts (GRBs) (i.e., Wang et al. 2015), can probe the nature of accelerating expansion.

SNe Ia are ideal standard candles to probe dark energy. But several effects can degrade their quality, such as the dust in light path (Avgoustidis et al. 2009), the possible intrinsic evolution in SN luminosity, gravitational lensing magnification (Holz 1998), peculiar velocity (Hui \& Greene 2006), and so on. These processes will degrade the standard candle usefulness of SNe Ia. Besides the above effects, Compton dimming due to free electrons deplete photons from standard candles by the (inverse) Compton scattering can cause systematic error for cosmological studies (Zhang 2008). These effects can degrade the evidence of accelerating expansion, or even mimic the dark energy behavior. So comprehensive study of the cosmic opacity is needed. Especially for the Wide Field Infrared Survey Telescope (WFIRST) era, which can detect more than 2000 SNe Ia (Green et al. 2012). If the cosmic opacity is not corrected, it will not increase statistical errors, but may also systematically bias the cosmological parameters.

Over the past several years, the cosmic distance duality (CDD) relation has been widely used to test the systematic errors and opacity in SNe Ia observations. The CDD relation reads (Etherington 1933; Ellis 2007)

$$
\frac{D_{L}}{D_{A}}(1+z)^{-2}=1
$$

where $D_{L}$ is the luminosity distance, and $D_{A}$ is the angular diameter distance. We must note that the cosmic opacity has no effect on the angular diameter $D_{A}$ (Weinberg 2008). It is valid for all cosmological models based on Riemannian geometry. The bases of this relation are that the number of photons is conservative and the photons travel along the null geodesics in a Riemannian spacetime (Ellis 2007). But the conservation of photons may be violated in a wide range of well-motivated models. In modern astronomy, the CDD relation plays a significant role. In order to test this relation, many works have been performed. For example, Bassett \& Kunz (2004) found a $2 \sigma$ violation of CDD relation using $D_{L}$ from SNe Ia and $D_{A}$ from FRIIb radio galaxies. The angular diameter from X-ray observations of galaxy clusters also has been used to probe the CDD relation (Holanda et al. 2011). Similar works have also been done by other authors (Meng et al. 2012; Goncalves et al. 2012). Räsänen et al. (2016) used CMB anisotropies to test the CDD relation. This relation is also applied extensively. Wang et al. (2012) and Cao et al. (2016) used the CDD relation to test the gas mass density profile of galaxy clusters. Evslin (2016) calibrated the distances of SNe Ia using the CDD relation.

A powerful method to study the opacity of the universe is using the standard candles to 
detect possible CDD deviations, such as SNe Ia and GRBs. For example, Avgoustidis et al. (2010) adopted a modified CDD relation

$$
D_{L}=D_{A}(1+z)^{2+\varepsilon}
$$

to constrain the cosmic opacity by combining the SNe Ia data (Kowalski et al. 2008) with the measurements of the Hubble expansion over redshift range $0<z<2$ (Stern et al. 2010). In the flat $\Lambda$ CDM model, they found $\varepsilon=-0.04_{-0.07}^{+0.08}(2 \sigma)$. In Avgoustidis et al. (2009), they marginalized over the parameter $H_{0}$ and used SNe Ia alone to constrain parameters $\Omega_{m}$ and $\varepsilon$. Li et al. (2013) presented some tests for the cosmic opacity with observational data including the Union 2.1 SNe Ia sample and galaxy cluster samples compiled by Filippis et al. (2005) and Bonamente et al. (2006). They found that an almost transparent universe is favored by the sample (Li et al. 2013). Basing on the validity of the Amati relation, Holanda et al. (2014) determined the cosmic opacity at high redshifts using GRBs, and found that a transparent universe is favored. Strong gravitational lensing systems are also used to probe the CDD realtion (Liao et al. 2015; Holanda et al. 2016).

Compared with previous papers, our paper has three advancements to this field. First, it must be noted that previous studies directly used the luminosity distances of SNe Ia, which are derived in the concordance cosmology (i.e., Avgoustidis et al. 2010; Li et al. 2013). The luminosity distances depend on the light-curve fitting parameters and cosmological models (Kowalski et al. 2008; Betoule et al. 2014). So the derived results are biased by the assumed cosmological model. Here, in order to avoid this problem, we perform a global fitting for the SNe Ia light-curve parameters, cosmological parameters and cosmic opacity. Second, we also investigate the cosmic opacity at high redshifts, where the fraction of electrons is evolving with redshift. The reionization process is considered. The cross section of Compton scattering for high-energy photons is also a function of redshfit. Third, the contribution from Compton scattering effect to the cosmic opacity is constrained for the first time. In this paper, we investigate the cosmic opacity with SNe Ia, long GRBs and Hubble parameter data. We pay special attention to the Compton scattering effect. This paper is organized as follows. In next section, we describe the cosmic opacity and Compton scatter extinction. In section 3, the observational data used in the statistical analysis are presented. The corresponding constraints on the cosmic opacity are given in section 4 . The paper is finished with a summary of the main results in the conclusion section.

\section{Cosmic opacity and Compton scattering}

Since photons can be scattered with free electrons and interstellar medium when travel from the source to the observer, the received photons number will be reduced. The distance 
modulus derived from standard candles will increase the systematic error. Any process reducing photon number would increase the luminosity distance of the source and dim its luminosity. Following Avgoustidis et al. (2009), we regard $\tau(z)$ as the opacity from the $z=0$ to the resource redshift due to extinction. Then, the received flux will decrease with a factor $e^{-\tau(z)}$. So the relation between observed luminosity distance $D_{L, o b s}$ and theoretic luminosity distance $D_{L, t h}$ is

$$
D_{L, o b s}=D_{L, t h} e^{\frac{\tau(z)}{2}} .
$$

The observed distance modulus is given by

$$
\mu_{o b s}(z)=\mu_{t h}(z)+2.5\left(\log _{10}(e)\right) \tau(z) .
$$

For flat FLRW cosmology, the distance modulus is

$$
D_{L, t h}(z)=(1+z) \frac{c}{H_{0}} \int_{0}^{z} \frac{d z}{E(z)},
$$

and

$$
E(z)=H(z) / H_{0}=\sqrt{\Omega_{m}(1+z)^{3}+\left(1-\Omega_{m}\right)(1+z)^{3+3 w}} .
$$

Combining equations (2) and (3), we obtain the exactly form of cosmic opacity

$$
\tau=2 \varepsilon \ln (1+z)
$$

\subsection{The optical depth of Compton scattering}

Compton scattering is the inelastic scattering of the photon by a charged free electron. The optical depth for Compton scattering is

$$
\tau_{c}(z)=\int \sigma_{T} n_{e}(z) d l=-(1+y) \sigma_{T} c \int_{0}^{z} n_{H}(z) Q_{H_{I I}}(z) \frac{d t}{d z} d z
$$

where $\sigma_{T}$ is the Thomson cross section, $n_{e}$ is the free electron density, $c$ is the light speed, and $z$ is the redshift. In the above equation, $n_{H}(z)=1.905 \times 10^{-7}(1+z)^{3} \mathrm{~cm}^{-3}$ is the hydrogen number density at redshift $z$, and $y$ is a factor which is introduced by including the ionization of helium. Because the reionization epoch contains both hydrogen and helium. The mass fractions of hydrogen and helium are $X=1-Y$ and $Y=0.24668$ (Planck Collaboration 2016), respectively. We assume that the helium was only ionized once. So we derive $y=$ $Y /(4 X) \approx 0.082$. $Q_{H_{I I}}(z)$ is defined as the volume filling fraction of ionized hydrogen, which can be calculated from the differential equation (Madau et al. 1999; Barkana \& Loeb 2001; Wang 2013)

$$
\dot{Q}_{\mathrm{H}_{\mathrm{II}}}=\frac{\dot{n}_{\gamma}(z)}{(1+y) n_{\mathrm{H}}(z)}-\alpha_{B} C(z)(1+y) n_{\mathrm{H}}(z) Q_{\mathrm{H}_{\mathrm{II}}} .
$$


In this equation, $\alpha_{B}=2.6 \times 10^{-13} \mathrm{~cm}^{-3} \mathrm{~s}^{-1}$ is the recombination coefficient for electron with temperature at about $10^{4} \mathrm{~K} . \dot{n}_{\gamma}(z)$ is the rate of ionizing photons escaping from the stars into the IGM, which can be derived from

$$
\dot{n}_{\gamma}(z)=(1+z)^{3} \frac{\dot{\rho}_{*(z)}}{m_{B}} N_{\gamma} f_{\text {esc }}
$$

where $(1+z)^{3}$ is used for converting the comoving density into the proper density, $\dot{\rho}_{*}(z)$ is the star formation rate (SFR), $m_{\mathrm{B}}$ is the baryon mass, $N_{\gamma}$ are the number of ionizing $\mathrm{UV}$ photons released per baryon, and $f_{\text {esc }}$ is the escape fraction of these photons from stars into IGM. The escape fraction is not well constrained from observations. $f_{\text {esc }} \leqslant 0.2$ is the average value suggested by Mao et al. (2007) and Robertson et al. (2015). Other similar value are reported. For instance, Razoumov \& Sommer-Larsen (2006) found that $f_{\text {esc }}$ evolves from $\sim 1-2$ percent at $z=2.39$ to $\sim 6-10$ percent at $z=3.6$ from star forming regions in young galaxies. Hayes et al. (2011) proposed a redshift evolution of $f_{\text {esc }}$. In this work, we take the value of $N_{\gamma}$ as $\sim 4000$ and the escape fraction $f_{\text {esc }} \simeq 0.1 . C \equiv\left\langle n_{H_{I I}}^{2}\right\rangle /\left\langle n_{H_{I I}}\right\rangle^{2}$ is the clumping factor of the ionized gas. Its value decreases with increasing redshifts from some numerical simulations (Gnedin \& Ostriker 1997; Shull et al. 2012) and semi-analytical studies (Madau et al. 1999; Chiu \& Ostriker 2000). Following Shull et al. (2012), we take

$$
C(z)= \begin{cases}2.9 & \text { if } \quad z<5 \\ 2.9\left(\frac{1+z}{6}\right)^{-1.1}, & \text { if } \quad z \geq 5\end{cases}
$$

$\dot{\rho}_{*(z)}$ is the SFR. The SFR derived by Wang (2013) is used. Then we can solve the differential equation (9) to obtain $Q_{H_{I I}}$. The result is shown in figure 1.

\subsection{The Compton scattering optical depth for SNe Ia}

Following $\mathrm{Hu}$ (1995) and Barkana \& Loeb (2001), the equation (8) with a constant ionization fraction can be expressed as

$$
\tau_{c}(z)=0.0461(1+y) Q_{H_{I I}}\left(1-Y_{p}\right) \frac{\Omega_{b} h}{\Omega_{m}}\left\{\left[1-\Omega_{m}+\Omega_{m}(1+z)^{3}\right]^{\frac{1}{2}}-1\right\},
$$

in the flat $\Lambda$ CDM model by neglecting the radiation term. At redshift range $0<z<3$, a constant ionization fraction $X_{e}(z)=1$ is adopted, which is reasonable for SNe Ia. The optical depth can increase the distance modulus with a relation $\Delta \mu=1.086 \tau$ from equation (4). Figure 2 shows the Compton scattering effect on the distance modulus. From this figure, we can see that the value of $\Delta \mu$ is increasing with redshift, and the Compton scattering dims the supernova flux by $0.003 \mathrm{mag}$ at $z=1$ and 0.01 mag at $z=2.35$, respectively. This 
dimming is too faint to rule out the existence of dark energy. However, its effect can not be negligible for future SNe Ia surveys plan such as WFIRST, which will measure $\sim 2700$ $\mathrm{SNe}$ Ia to $z \sim 1.7$. For future surveys, the major statistical uncertainty is the SN intrinsic fluctuations. With the SNe Ia number $N$, the intrinsic fluctuations are reduced to a level of $\sigma_{\mu} / \sqrt{N}$ mag, where $\sigma_{\mu}$ is the intrinsic dispersion in SN luminosity. It means that the Compton dimming effect must be corrected. Otherwise the induced systematic errors would be comparable to the statistical errors. From above analysis, we conclude that the Compton scattering can be correctable, as discussed by Zhang (2008).

\subsection{The Compton scattering dimming for GRBs}

The photons emitted from GRBs are different from those from SNe Ia. First, the energy of the GRB photons are much more energetic. At high energies, cross section of the Compton scattering is suppressed. So more photons can escape from scattering to the observer. Second, GRBs can be observed at high redshifts. High-energy photons have much more probability to interact with free electrons. The optical depth of Compton scattering for high-energy photons can be written as

$$
\tau_{c}(z)=-(1+y) c \int_{0}^{z} \sigma(x) n_{H}(z) Q_{H_{I I}}(z) \frac{d t}{d z} d z
$$

where $\sigma(x)=\sigma\left(E_{0}(1+z) / m_{e} c^{2}\right)$ is given by the Klein-Nishina formula Rybicki \& Lightman 1976)

$$
\sigma(x)=\frac{3}{4} \sigma_{T}\left[(1+x) \frac{2 x(1+x) /(1+x)-\ln (1+2 x)}{\mathrm{x}^{3}}+\frac{\ln (1+2 x)}{2 x}-\frac{1+3 x}{(1+2 x)^{2}}\right] .
$$

Here, $E_{0}$ is the observed energy of $\gamma$-ray photons. The future SOVM (Space-based multiband astronomical Variable Objects Monitor) mission, will detect some GRBs at $z>10$ (Wei et al. 2016). At these high-redshifts, the hydrogen is not completely ionized. The parameter $Q_{H_{I I}}$ is a constant in equation (13), and the reionization process must be considered. We use the reionization process described in Section 2.1 to calculate the optical depth. The systematic shift in distance modulus $\Delta \mu$ due to Compton scattering is shown in figure 3. It is obvious that the effect of Compton scattering for low-energy photons is significant, because the cross section is suppressed for high-energy photons. The evolution of $\Delta \mu$ becomes flat at high redshifts, due to few free electrons from reionization. The $\Delta \mu$ caused by Compton dimming increases with reshift. Its value can reach to 0.01-0.04 mag, which is smaller than the intrinsic error of GRB distance (Wang et al. 2016). So we can ignore it if the number of GRBs is less than 100 and the redshift of GRB is not very high. 
However, if more than 100 high-redshift long GRBs will be used to study cosmology, the Compton dimming is non-negligible.

\section{Data set}

In this section, we will show the data sets. These data sets will be used to constrain the cosmic opacity and cosmological parameters. Unlike previous works, we try to global fit the SNe Ia light-curve parameters, cosmological parameters and the cosmic opacity.

\section{1. $\quad \mathrm{SNe}$ Ia sample}

In this work, we use 740 SNe Ia from the "joint light-curve analysis" sample compiled by Betoule et al. (2014). The redshift range is from 0.01 to 1.299. This sample includes SNe Ia from different surveys.In their work, they regard the possible extinction as systematic uncertainty. In order to avoid the effect of cosmological model effect, the parameters of the SNe Ia light-curve, cosmological parameters and the cosmic opacity are fitting simultaneously. Therefore, only the statistical error which from error propagation of light-curve fitting uncertainties and the variation of magnitudes caused by the intrinsic variation in SN magnitude are needed to consider in our work. The possible extinctions are all regarded as cosmic opacity. The distance modulus is written as

$$
\mu=m_{B}^{\star}-\left(M_{B}-\alpha \times X_{1}+\beta \times C\right),
$$

where $m_{B}^{\star}$ is the observed peak magnitude in rest-frame $B$ band. $\alpha$ and $\beta$ are nuisance parameters which describe the stretch-luminosity and color-luminosity relations, reflecting the well-known broader-brighter and bluer-brighter relations, respectively. The nuisance parameter $M_{B}$ represents the absolute magnitude of a fiducial SNe Ia and is found to depend on the properties of host galaxies, e.g., the host stellar mass $\left(M_{\text {stellar }}\right)$. Here, we follow the

procedure in Conley et al. (2011) to approximately correct for this effect by a simple step function:

$$
M_{B}= \begin{cases}M_{B}^{1} & \text { if } M_{\text {stellar }}<10^{10} M_{\odot} \\ M_{B}^{1}+\Delta_{M} & \text { otherwise }\end{cases}
$$

\subsection{GRB sample}

For GRBs, we use the GRB data given in Wang et al. (2016). They use the $E_{i s o}-E_{p}$ correlation Amati et al. (2002) to build the Hubble diagram. Wang et al. (2016) combine 
their 42 GRBs and 109 GRBs from Amati et al. (2008) and Amati et al. (2009). The $E_{i s o^{-}} E_{p}$ correlation can be written as

$$
\log \frac{E_{i s o}}{\operatorname{erg}}=c+d \log \frac{E_{p}}{\mathrm{kev}},
$$

where parameters $c$ and $d$ are free parameters, $E_{i s o}$ is the isotropic equivalent energy, and $E_{p}$ is the peak energy of $\nu F_{\nu}$ spectrum, which has been corrected into the cosmological rest frame. In their work, they calibrate 90 high-redshift GRBs in the redshift range from 1.44 to 8.1 with a fixed value of $H_{0}$. We constrain the cosmological parameters and the cosmic opacity use this sub-sample (Wang et al. 2016). In order to consider the effect of Compton dimming, we show the value of $\Delta \mu$ for this sample as dots in figure 3 , which is derived from equations (4) and (13). The error bar is due to the uncertainty of the observational peak energy of GRBs. The value of $\Delta \mu$ caused by Compton dimming is far less than the top black dash line. Therefore, we can ignore this effect in following work.

\section{3. $H(z)$ sample}

The 19 Hubble parameter data given in Simon et al. (2005), Stern et al. (2010), and Moresco et al. (2012) are used in this work. The redshift range of these Hubble parameters is from 0.10 to 1.75 . Because $H_{0}$ will affect the final results, we regard $H_{0}$ as a free parameter.

\section{Results}

The maximum likelihood analysis is used to constrain the parameters. The $\chi^{2}$ fitting expression is

$$
\chi^{2}=\sum_{i}^{n} \frac{\left[\mu_{o b s}-\mu_{t h}\left(z_{i}\right)-1.086 \tau\left(z_{i}\right)\right]}{\sigma_{\mu}^{2}}+\chi_{H(z)}^{2}
$$

In our analysis, we adopt the cosmic opacity from equation (7). The parameter $\varepsilon$ is regarded as a constant. For data of SNe Ia and $H(z)$, The $\mu_{o b s}$ for SNe Ia is written as equation (15). $\sigma_{\mu}^{2}=\sigma_{\mu, \text { stat }}^{2}+\sigma_{\mu, \text { sys }}^{2}$ is the distance modulus uncertainty. $\sigma_{\mu, \text { stat }}^{2}$ is the propagated error from the covariance matrix of the light-curve fitting, and $\sigma_{\mu, s y s}$ is the systematic error due to the intrinsic variation in SNe Ia magnitude. The value $\sigma_{\mu, s y s}$ is calculated in Betoule et al. (2014), which is not depend on a specific choice of cosmological model. $\mu_{t h}$ is the theoretic distance modulus which is depend on cosmological model. $\chi_{H(z)}^{2}$ is the $\chi^{2}$ fitting of Hubble parameter data, which can be calculated by

$$
\chi_{H(z)}^{2}=\sum_{i}^{m} \frac{\left[H_{o b s}\left(z_{i}\right)-H_{t h}\left(z_{i}\right)\right]^{2}}{\sigma_{H_{o b s}}^{2}},
$$


where the $H_{o b s}$ is the observation value, $H_{t h}$ is the theoretic Hubble expansion rate related to cosmological model, and $\sigma_{H_{o b s}}$ is the error of $H_{o b s}$. For the GRB data, because Wang et al. (2016) calibrated the distance moduli by fixing $H_{0}=67.8 \mathrm{~km} \mathrm{~s}^{-1} \mathrm{Mpc}^{-1}$, so the value of $H_{0}$ is fixed when using the GRB data. We use the Markov chain Monte Carlo (MCMC) method to fit the parameters of the SNe Ia light-curve, cosmological parameters and the cosmic opacity simultaneously. Our program is based on the public emcee Python module (Foreman-Mackey et al. 2013). The algorithm of emcee has several advantages over traditional MCMC methods and it has excellent performance as measured by the autocorrelation time.

\subsection{Flat $\Lambda \mathrm{CDM}$}

In this model, the equation of state $w$ in equation (6) has a fixed value with $w=-1$. When using the SNe Ia $+H(z)$ data, the free parameters are $M_{B}, \alpha, \beta, \Delta M, H_{0}, \Omega_{m}$, and $\varepsilon$. We use the emcee Python module to fit these parameters simultaneously. The fitting result is shown in figure 4. The 2-D regions and $1-\mathrm{D}$ marginalized distributions with $1 \sigma$ and $2 \sigma$ contours for the parameters $M_{B}, \alpha, \beta, \Delta M, H_{0}, \Omega_{m}$, and $\varepsilon$ are shown. The fitting results of parameters are presented in table I. The value of $\varepsilon$ is $0.0226_{-0.0451}^{+0.0403}$, which indicates an almost transparent universe. For GRB $+H(z)$ data, there are only two free parameters: $\Omega_{m}$ and $\varepsilon$. The fitting results are shown in figure 5 and table I. The value $\varepsilon=0.00718_{-0.0492}^{+0.0486}$ also supports a transparent universe.

\subsection{Flat XCDM}

In a flat XCDM cosmology, the parameter $w$ in equation(6) is a free parameter. When using the SNe Ia $+H(z)$ data, the free parameters are $M_{B}, \alpha, \beta, \Delta M, H_{0}, \Omega_{m}, w$ and $\varepsilon$. Using the same method as above, we can perform the simultaneously fitting of these parameters. The 2-D regions and 1-D marginalized distributions with $1 \sigma$ and $2 \sigma$ contours for the parameters $M_{B}, \alpha, \beta, \Delta M, H_{0}, \Omega_{m}$, and $\varepsilon$ are shown in figure 6 and table I. They are shown in the fourth and the last column of table I. The value of $\varepsilon=$ is $0.0517_{-0.0659}^{+0.0617}$. For $\mathrm{GRB}+H(z)$ data, there are three parameters: $\Omega_{m}, w$ and $\varepsilon$. The fitting results are shown in figure 7 and table I. The value $\varepsilon=0.0718_{-0.0491}^{+0.0497}$ also indicates a transparent universe. 


\subsection{Considering the effect of Compton dimming}

Because the effect of Compton dimming can be estimated, the residual opacity can be derived. We try to eliminate the known opacity due to Compton scattering, and explore the contribution by unknown part. In the equation (12), we get the optical depth of Compton scattering of SNe Ia. After subtracting the optical depth of Compton scattering from total cosmic opacity, we repeat the above analysis to obtain the residual opacity $\tau_{r}$. In flat $\Lambda$ CDM model, the results from $\mathrm{SNe} \mathrm{Ia}+H(z)$ are shown in figure 8 , which gives $\varepsilon=0.0212_{-0.0413}^{+0.0382}$. Constraints on parameters are shown in the column 3 of table I. Similar results are also shown in figure 8 and column 5 of table I for XCDM model. Comparing the second and third columns in table I, it can be seen that the effect of Compton scattering can cause about $5 \%$ cosmic opacity in $\Lambda$ CDM model. For XCDM model, a similar percentage is found. So Compton scattering can contribute about a few percent of cosmic opacity. It's obvious that the sample supports an almost transparent universe for both cosmological models.

\section{Conclusions and discussion}

In this paper, we use the latest observations, including SNe Ia from JLA sample and Hubble parameters, to study cosmic opacity. The effect of Compton scattering on standard candles is also considered. The extinction due to Compton scattering can be correctable in future SNe Ia survey. In order to avoid the cosmological dependence of SNe Ia luminosity distances, a joint fitting of the SNe Ia light-curve parameters, cosmological parameters and opacity is used. In order to explore the cosmic opacity at high redshifts, the latest gammaray bursts (GRBs) are used. Because some instruments will detect high-redshift GRBs in future, the reionization process must be considered for Compton scattering. The result shows that the Compton dimming effect is less than the systematic error for GRBs at present. However, if more than 100 high-redshift long GRBs are observed and used to constrain cosmological parameters, the Compton dimming is non-negligible. The results support an almost transparent universe at $z<1.5$ for JLA SNe Ia and $H(z)$ data. In the redshift range $1.5<z<8.1$, we study the cosmic opacity through luminosity distances of GRBs. The flat $\Lambda \mathrm{CDM}$ model and the flat XCDM model are considered. We find that the effect

of Compton scattering can cause about $5 \%$ cosmic opacity in both models. The current observations support an almost transparent universe for both cosmological models at a large redshift range. 


\section{Acknowledgements}

We thank the anonymous referee for useful comments. This work is supported by the National Basic Research Program of China (973 Program, grant No. 2014CB845800), the

National Natural Science Foundation of China (grants 11422325 and 11373022), and the Excellent Youth Foundation of Jiangsu Province (BK20140016).

\section{REFERENCES}

Amati, L., Frontera, F., Tavani, M., et al., 2002, A\&A, 390, 81

Amati, L., Guidorzi, C., Frontera, F., et al. 2008, MNRAS, 391, 577

Amati L., Frontera, F., \& Guidorzi, C., 2009, A\&A, 508, 173

Avgoustidis, A., Burrage, C., Redondo, J., Verde, L. \& Jimenez, R., 2009, JCAP, 06, 012.

Avgoustidis, A., Burrage, C., Redondo, J., Verde, L. \& Jimenez, R., 2009, JCAP, 10, 024

Barkana, R., \& Loeb, A. 2001, PhR, 349, 125

Betoule, M., et al., 2014, A\&A, 568, A22

Bassett, B., A., \& Kunz, M., 2004, PRD, 69, 101305

Cao, S., et al., 2016, MNRAS, 457, 281

Capozziello, S., 2002, Int. J. Mod. Phys. D, 11, 483

Chiu, W. A. \& Ostriker, J. P. 2000, ApJ, 534, 507

Combes, F., 2004, New Astronomy Rev, 48, 583

Conley, A., et al., 2011, ApJS, 192, 1

Drell, P. S., Loredo, T. J., \& Wasserman, I., 2000, ApJ, 530, 593

Eisenstein, D. J., et al. 2005, ApJ, 633, 560

Ellis, G. F. R., 2007, Gen. Relativ. Gravit, 39, 1047

Etherington, I. M. H., 1933, Philos. Mag, 15, 761

Evslin, J., 2016, PDU, 14, 57 
Foreman-Mackey, D., Hogg, D.W., Lang, D., Goodman, J., 2013, PASP, 125, 306

Gnedin, N. Y., \& Ostriker, J.P., 1997, ApJ. 486, 581

Goncalves,R. S., Holanda, R. F. L., \& Alcaniz, J. S., 2012, MNRAS, 420, L43

Green, J., et al., 2012, arXiv: 1208.4012

Filippis, E. De., Sereno, M., Bautz W., \& Longo, G., 2005, ApJ, 625, 108

Hayes, M., Schaerer, D., Östlin, G., et al. 2011, ApJ, 730, 8

Holanda, R. F. L., Lima, J. A. S., \& Ribeiro, M. B., 2011, A\&A, 528, L14

Holanda, R. F. L. \& Busti, V. C., 2014, Phys. Rev. D, 89, 103517

Holanda, R. F. L. \& Busti, V. C., 2016, JCAP, 89, 103517

Holz, D. E. 1998, ApJ, 506, L1

Hu, W., 1995. Ph.D. Thesis, astro-ph/9508126

Hui, L. \& Greene, P., 2006, PRD, 73, 123526

Jimenez, R., Verde, L., Treu, T., Stern, D., 2003, ApJ, 593, 622

Kowalski et al. 2008, ApJ, 686, 749

Li, Z. X., Wu, P. X., Yu, H. W., \& Zhu, Z. H., 2013, PRD, 87, 103013

Liao, K., Li, Z. X., Cao, S., Biesiada, M., Zheng, X. G., \& Zhu, Z. H., 2015, ApJ, 822, 2

Bonamente, M., Joy, M. K., LaRoque, S. J., Carlstrom, J.E., Reese, E. D., \& Dawson, K. S., 2006, ApJ, 647, 25.

Madau, P., Haardt, F., \& Rees, M. J. 1999, ApJ, 514, 648

Mao, J., Lapi, A., Granato, G. L., de Zotti, G., \& Danese, L. 2007, ApJ, 667, 655

Meng, X. L., Zhang, T. J., Zhan, Hu., \& Wang, Xin., 2012, ApJ, 745, 98

Moresco, M. et al., 2012, JCAP, 08, 006

Perlmutter, S., et al. 1999, ApJ, 517, 565

Planck Collaboration, Ade, P. A. R., et al., 2016, A\&A, 594, A13 
Räsänen, S., Valiviita, J. \& Kosonen, V., 2016, JCAP, 04, 050

Razoumov, A. O., \& Sommer-Larsen, J., 2006, ApJ, 651, L89

Riess, A. G., et al. 1998, AJ, 116, 1009

Rybicki, G.B. \& Lightman, A.P., 1979, Radiative Processes in Astrophysics. John Wiley \& Sons, Inc.

Robertson, B. E., Ellis, R. S., Furlanetto, S. R., \& Dunlop, J. S., 2015, ApJL, 802, L19

Shull J. M., Harness A., Trenti M. \& Smith B. D., 2012, ApJ, 747, 100

Simon, J., Verde, L., \& Jimenez, R., 2005, PRD, 71, 123001

Spergel, D. N., et al. 2003, ApJS, 148, 175

Stern, D., Jimenez, R., Verde, L., Stanford, S. A., \& Kamionkowski, M., 2010, ApJS, 188, 280

Tan, W. W., Wang, F. Y., \& Cheng, K. S., 2016, ApJ, 829, 29

Wang, F. Y., 2013, A\&A, 556, A90

Wang, F. Y., Dai, Z. G. \& Liang, E. W., 2015, New Astro. Rev., 67, 1

Wang, J. S., Wang, F. Y., Cheng, K. S., \& Dai, Z. G., 2016, A\&A, 13, 9

Wang, X., Meng, X. L., Huang, Y. F., \& Zhang, T. J., 2012, A\&A, 585, A68

Wei, J., et al., 2016, arXiv:1610.06892, SVOM white book

Weinberg, S., 2008, Cosmology., Oxford University Press

Zhang, P. J., 2008, ApJ, 682, 721 


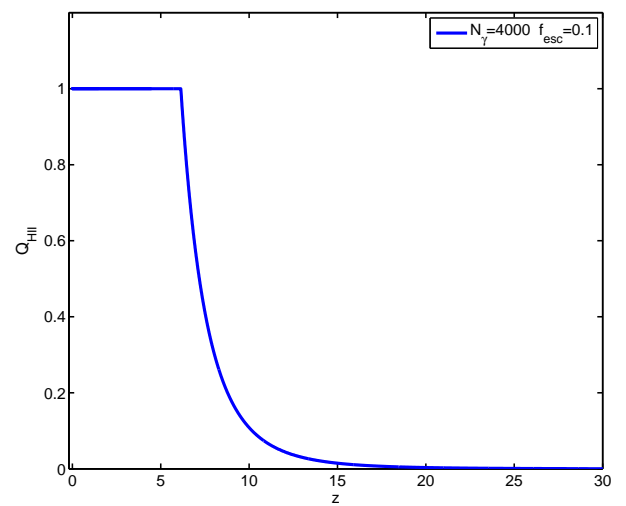

Fig. 1. $-H_{I I}$ filling factor $Q_{H_{I I}}$ as a function of redshift calculated for the $f_{\text {esc }}=0.1$ and $N_{\gamma}=4000$.

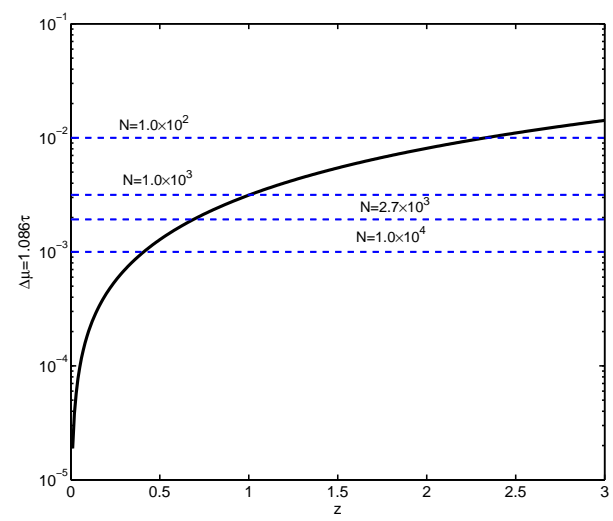

Fig. 2.- Systematic shift in the distance modulus $\mu$ caused by Compton scattering (solid line). The dimming is $0.3 \%$ in flux at $z=1$ and $1 \%$ at $z=2.35$ with Compton dimming effect. The statistical errors for 100, 1000, 2700 and 10000 SNe are shown as the dash lines. We adopt intrinsic dispersion $\sigma_{\mu}=0.1 \mathrm{mag}$ for SNe Ia. 


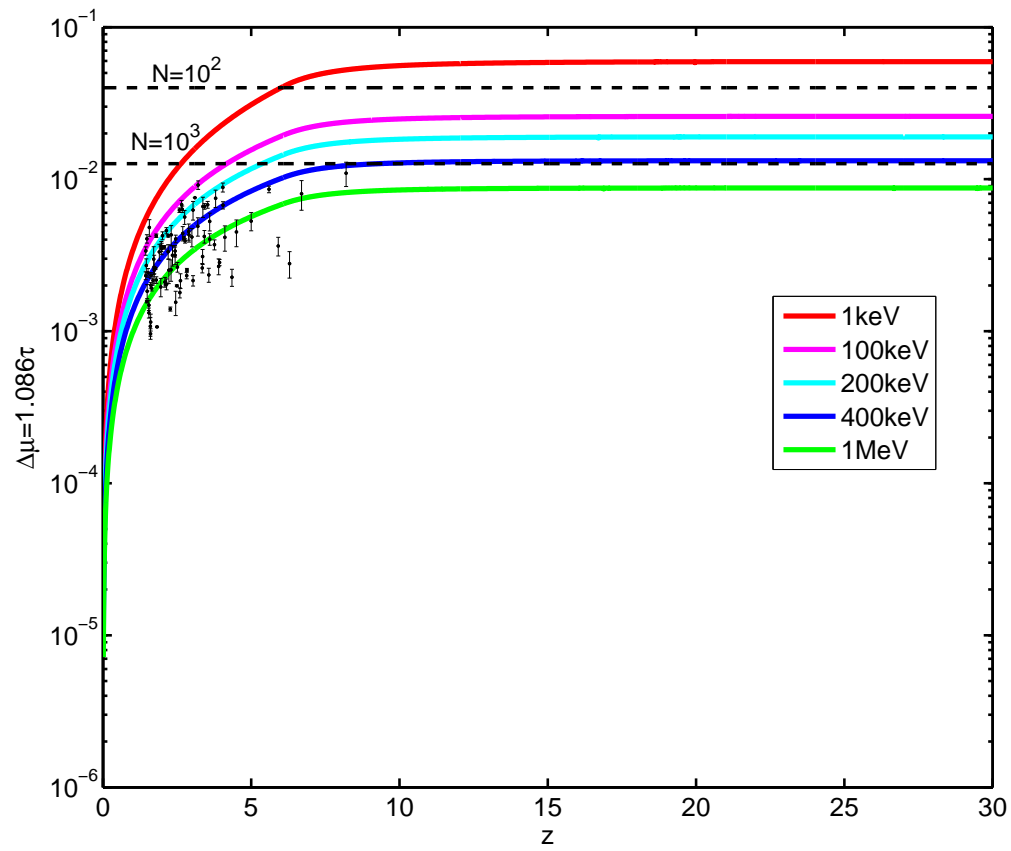

Fig. 3.- The systematic shift in distance moduli $\Delta \mu$ for GRBs. We consider the ionization fraction as a function of redshift. The Compton scattering cross section is energy dependent, because the photons of GRBs are energetic. The statistical errors for 100 and 1000 GRBs are shown by the dash lines, respectively. We adopt intrinsic dispersion $\sigma_{\mu}=0.4 \mathrm{mag}$ for GRBs. The black dots are the $\Delta \mu$ of observed GRBs caused by Compton dimming. 


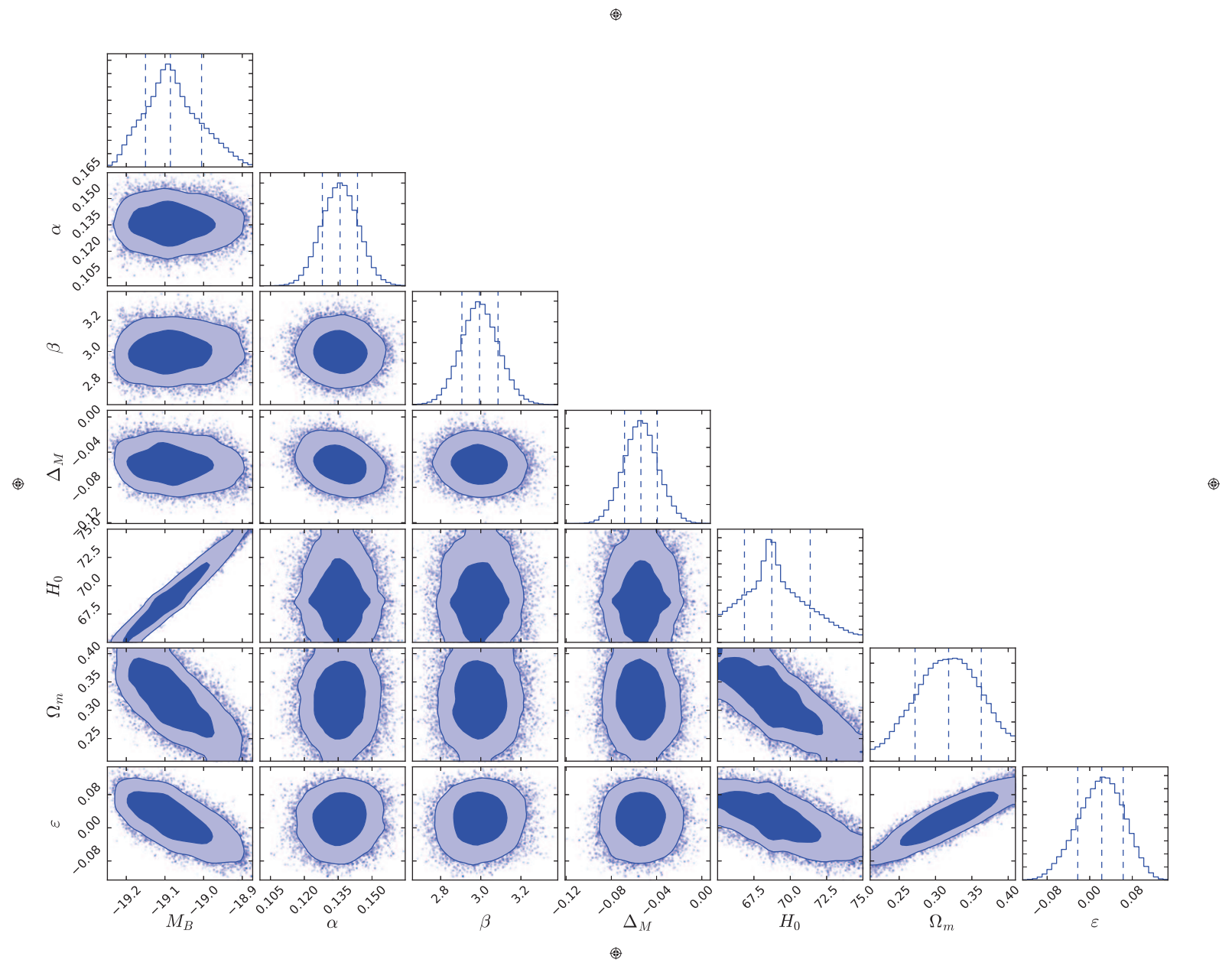

Fig. 4. - In $\Lambda$ CDM model, the 2-D regions and 1-D marginalized distributions with $1 \sigma$ and $2 \sigma$ contours for the parameters $M_{B}, \alpha, \beta, \Delta_{M}, H_{0}, \Omega_{m}$, and $\varepsilon$ using SNe Ia $+H(z)$. 
$\bigoplus$

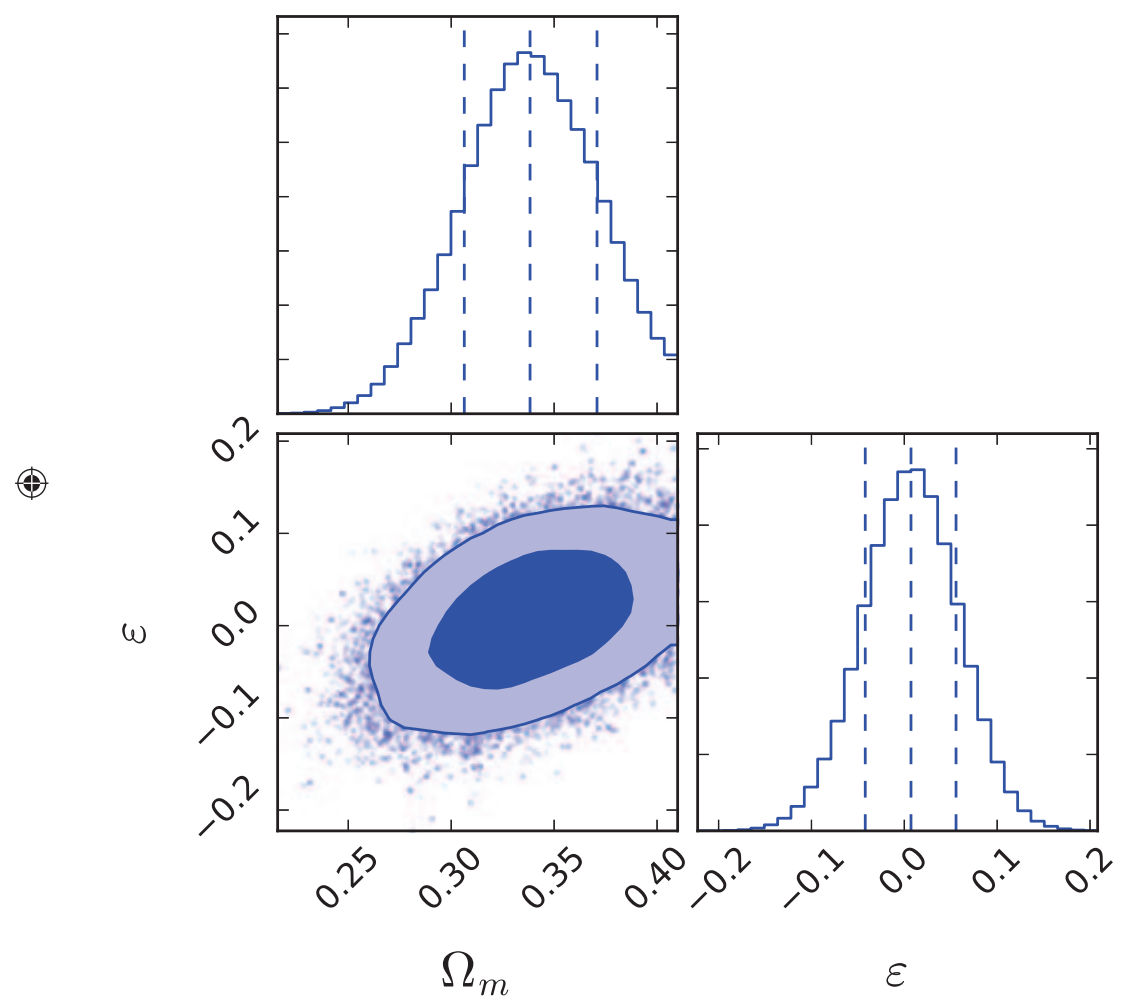

$\oplus$

Fig. 5.- The 2 - D regions and 1 - D marginalized distributions with $1 \sigma$ and $2 \sigma$ contours for the parameters $\Omega_{m}, \varepsilon$ using GRBs $+H(z)$ in $\Lambda$ CDM model. 


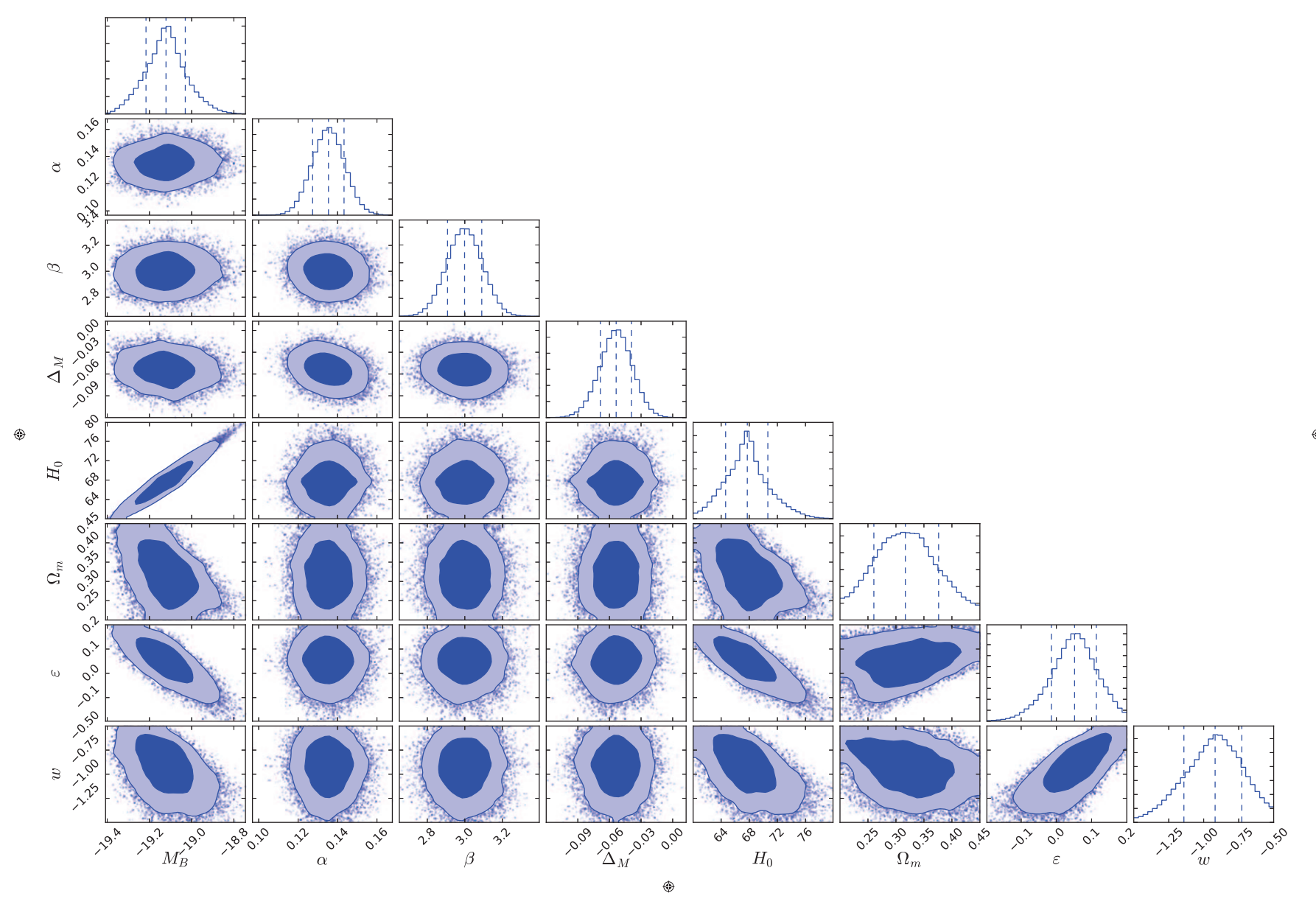

Fig. 6. - In XCDM model, 2 - D regions and 1 - D marginalized distributions with $1 \sigma$ and $2 \sigma$ contours for the parameters $M_{B}, \alpha, \beta, \Delta_{M}, H_{0}, \Omega_{m}, \varepsilon$ using SNe Ia $+H(z)$. 


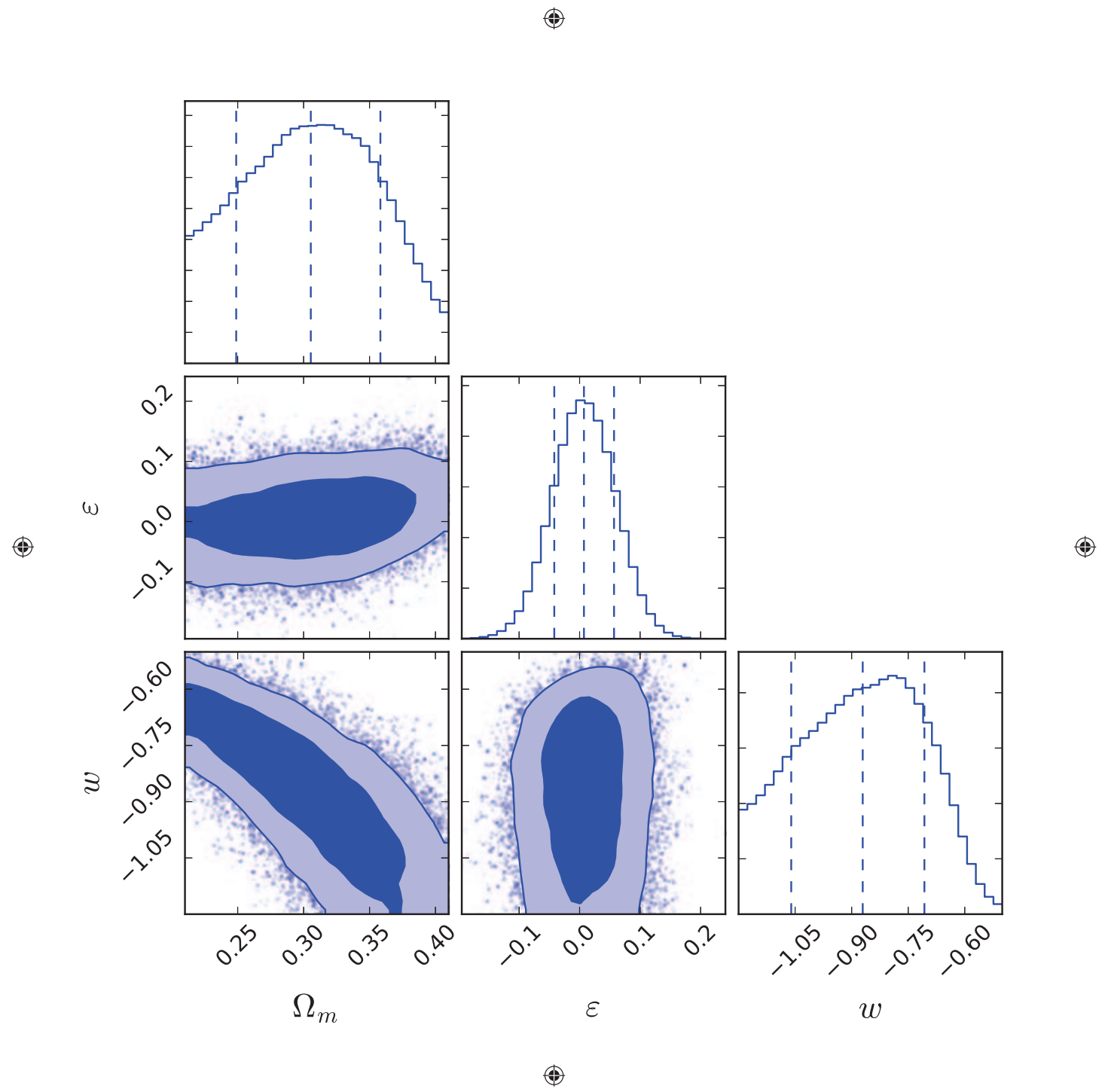

Fig. 7.- In XCDM model, 2 - D regions and 1 - D marginalized distributions with $1 \sigma$ and $2 \sigma$ contours for the parameters $\Omega_{m}, \varepsilon$ using GRBs $+H(z)$. 
(4)



Fig. 8.- In $\Lambda$ CDM model, 2 - D regions and 1 - D marginalized distributions with $1 \sigma$ and $2 \sigma$ contours for the parameters $M_{B}, \alpha, \beta, \Delta_{M}, H_{0}, \Omega_{m}$, and $\varepsilon$ using SNe Ia $+H(z)$ after subtracting the effect of Compton scattering. 


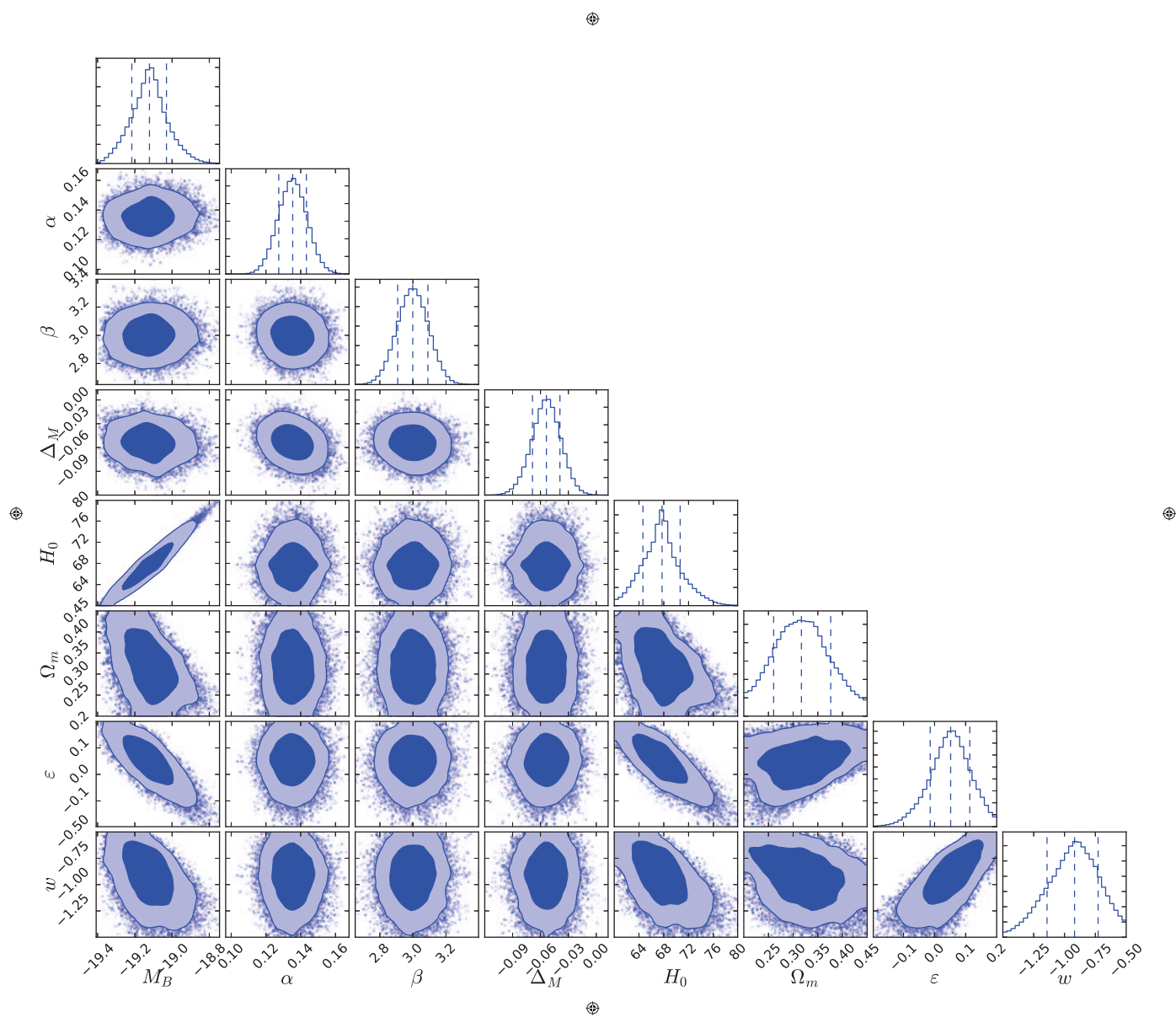

Fig. 9.- In XCDM model, 2 - D regions and 1 - D marginalized distributions with $1 \sigma$ and $2 \sigma$ contours for the parameters $M_{B}, \alpha, \beta, \Delta_{M}, H_{0}, \Omega_{m}$, and $\varepsilon$ using SNe Ia $+H(z)$ after subtracting the effect of Compton scattering. 
Table 1: Constraints on $\varepsilon$, light-curve parameters and cosmological parameters with $1 \sigma$ confidence level in different models.

\begin{tabular}{|c|c|c|c|c|c|c|}
\hline Model & \multicolumn{3}{|c|}{$\Lambda$ CDM } & \multicolumn{3}{c|}{ XCDM } \\
\hline data set & \multicolumn{2}{|c|}{$\mathrm{H}(\mathrm{z})+\mathrm{SNe}$ Ia } & $\mathrm{H}(\mathrm{z})+\mathrm{GRBs}$ & \multicolumn{2}{c|}{$\mathrm{H}(\mathrm{z})+\mathrm{SNe}$ Ia } & $\mathrm{H}(\mathrm{z})+\mathrm{GRBs}$ \\
\hline$\tau(z)$ & $2 \varepsilon \ln (1+z)$ & subtracting $\tau_{c}$ & $2 \varepsilon \ln (1+z)$ & $2 \varepsilon \ln (1+z)$ & 2 subtracting $\tau_{c}$ & $2 \varepsilon \ln (1+z)$ \\
\hline $\mathrm{a}$ & $0.136_{-0.00783}^{+0.00772}$ & $0.136_{-0.00756}^{+0.00749}$ & $/$ & $0.135_{-0.00803}^{+0.00790}$ & $0.136_{-0.00773}^{+0.00780}$ & $/$ \\
\hline $\mathrm{b}$ & $2.994_{-0.0878}^{+0.0923}$ & $3.000_{-0.0908}^{+0.0937}$ & $/$ & $2.999_{-0.0907}^{+0.0916}$ & $3.002_{-0.0893}^{+0.0089}$ & $/$ \\
\hline$M_{B}$ & $-19.086_{-0.0643}^{+0.0807}$ & $-19.088_{-0.0638}^{+0.0756}$ & $/$ & $-19.121_{-0.0948}^{+0.0913}$ & $-19.113_{-0.0854}^{+0.0868}$ & $/$ \\
\hline$\Delta_{M}$ & $-0.0539_{-0.0145}^{+0.0145}$ & $-0.0539_{-0.0144}^{+0.0147}$ & $/$ & $-0.0536_{-0.0150}^{+0.0146}$ & $-0.0543_{-0.0152}^{+0.0142}$ & $/$ \\
\hline$H_{0}$ & $68.732_{-1.884}^{+2.641}$ & $68.715_{-1.929}^{+2.474}$ & 67.8 fixed & $67.718_{-3.035}^{+2.923}$ & $67.857_{-2.738}^{+2.917}$ & 67.8 fixed \\
\hline$\Omega_{m}$ & $0.318_{-0.0462}^{+0.0449}$ & $0.320_{-0.0448}^{+0.0412}$ & $0.338_{-0.0319}^{+0.0325}$ & $0.317_{-0.0563}^{+0.0594}$ & $0.308_{-0.0553}^{+0.0536}$ & $0.305_{-0.0569}^{+0.0528}$ \\
\hline$w$ & $/$ & $/$ & $/$ & $-0.919_{-0.223}^{+0.191}$ & $-0.906_{-0.205}^{+0.200}$ & $-0.871_{-0.190}^{+0.164}$ \\
\hline$\varepsilon$ & $0.0226_{-0.0451}^{+0.0403}$ & $0.0212_{-0.0413}^{+0.0382}$ & $0.00718_{-0.0492}^{+0.0486}$ & $0.0517_{-0.0659}^{+0.0617}$ & $0.0490_{-0.0654}^{+0.0590}$ & $0.0718_{-0.0491}^{+0.0497}$ \\
\hline
\end{tabular}

